\title{
Does Coffee Intake Reduce Postoperative Ileus After Laparoscopic Elective Colorectal Surgery?: A Prospective, Randomized Controlled Study: The Coffee Study
}

\author{
Hasler-Gehrer, Simone ; Linecker, Michael ; Keerl, Andreas ; Slieker, Juliette ; Descloux, Alexandre ; \\ Rosenberg, Robert ; Seifert, Burkhardt ; Nocito, Antonio
}

\begin{abstract}
BACKGROUND Postoperative ileus after colorectal surgery is a frequent problem that significantly prolongs hospital stay and increases perioperative costs. OBJECTIVE The aim was to evaluate the effect of standardized coffee intake on postoperative bowel movement after elective laparoscopic colorectal resection. DESIGN This is a prospective randomized controlled trial that was conducted between September 2014 and December 2016. SETTINGS This study was performed in a public cantonal hospital in Switzerland with accreditation for colon and rectum cancer surgery. PATIENTS Patients who underwent elective colorectal surgery were included. INTERVENTIONS Patients were randomly assigned either to the intervention group receiving coffee or the control group receiving tea. A total of $150 \mathrm{~mL}$ of the respective beverage was drunk 3 times per day every postoperative day until discharge. MAIN OUTCOME MEASURES The primary end point was time to first bowel movement. Secondary end points included the use of laxative, insertion of a nasogastric tube, length of hospital stay, and postoperative complications. RESULTS A total of 115 patients were randomly assigned: 56 were allocated to the coffee group and 59 to the tea group. After coffee intake, the first bowel movement occurred after a median of 65.2 hours versus 74.1 hours in the control group (intention-to-treat analysis; $\mathrm{p}=0.008$ ). The HR for earlier first bowel movement after coffee intake was $1.67(\mathrm{p}=0.009)$. In the per-protocol analysis, hospital stay was shorter in the coffee group $(6 \mathrm{~d}$ in the coffee group vs $7 \mathrm{~d}$ in the tea group; $\mathrm{p}=0.043)$. LIMITATIONS The rate of protocol violation, mostly coffee consumption in the tea arm, was relatively high, even if patients were clearly instructed not to consume coffee if they were in the tea arm. CONCLUSIONS Coffee intake after elective laparoscopic colorectal resection leads to faster recovery of bowel function. Therefore, coffee intake represents a simple and effective strategy to prevent postoperative ileus. See Video Abstract at http://links.lww.com/DCR/A955.
\end{abstract}

DOI: https://doi.org/10.1097/DCR.0000000000001405

Posted at the Zurich Open Repository and Archive, University of Zurich

ZORA URL: https://doi.org/10.5167/uzh-171259

Journal Article

Published Version

Originally published at:

Hasler-Gehrer, Simone; Linecker, Michael; Keerl, Andreas; Slieker, Juliette; Descloux, Alexandre; Rosenberg, Robert; Seifert, Burkhardt; Nocito, Antonio (2019). Does Coffee Intake Reduce Postoperative Ileus After Laparoscopic Elective Colorectal Surgery?: A Prospective, Randomized Controlled Study: The Coffee Study. Diseases of the colon and rectum, 62(8):997-1004. 
DOI: https://doi.org/10.1097/DCR.0000000000001405 


\title{
Does Coffee Intake Reduce Postoperative Ileus After Laparoscopic Elective Colorectal Surgery? : A Prospective, Randomized Controlled Study: The Coffee Study
}

\author{
Simone Hasler-Gehrer, M.D. ${ }^{1}$ Michael Linecker, M.D. ${ }^{1,2}$ • Andreas Keerl, M.D. ${ }^{1}$ \\ Juliette Slieker, M.D. ${ }^{1} \bullet$ Alexandre Descloux, M.D. ${ }^{1}$ Robert Rosenberg, M.D. ${ }^{3}$ \\ Burkhardt Seifert, Ph.D. ${ }^{4}$ Antonio Nocito, M.D. ${ }^{1}$ \\ 1 Department for General, Visceral and Vascular Surgery, Kantonsspital Baden, Baden, Switzerland \\ 2 Department of Surgery and Transplantation, University Hospital Zurich, Zurich, Switzerland \\ 3 Department for General, Visceral, Thoracic and Vascular Surgery, Kantonsspital Baselland, Liestal, Switzerland \\ 4 Department of Biostatistics at Epidemiology, Biostatistics and Prevention Institute, University Zurich, Zurich, Switzerland
}

BACKGROUND: Postoperative ileus after colorectal surgery is a frequent problem that significantly prolongs hospital stay and increases perioperative costs.

OBJECTIVE: The aim was to evaluate the effect of standardized coffee intake on postoperative bowel movement after elective laparoscopic colorectal resection.

DESIGN: This is a prospective randomized controlled trial that was conducted between September 2014 and December 2016.

SETTINGS: This study was performed in a public cantonal hospital in Switzerland with accreditation for colon and rectum cancer surgery.

PATIENTS: Patients who underwent elective colorectal surgery were included.

Funding/Support: None reported.

Financial Disclosure: None reported.

Presented at the Swiss Visceral Surgeon Congress, Lausanne, Switzerland, September 14 to 15, 2017; the meeting of the European Society of Coloproctology (as a part of the Six Best Papers session), Berlin, Germany, September 20 to 22, 2017; the meeting of the German Coloproctology Congress, Munich, Germany, March 15 to 17, 2018 (where we won the Jens J. Kirsch prize); and at the Swiss Surgeon Congress, Basel, Switzerland, May 16 to 18,2018 (as a part of the award winner session).

Correspondence: Antonio Nocito, M.D., Department of General, Visceral and Vascular Surgery, Kantonsspital Baden, 5404 Baden, Switzerland. E-mail: antonio.nocito@ksb.ch

Dis Colon Rectum 2019; 62: 00-00

DOI: 10.1097/DCR.0000000000001405

(c) The ASCRS 2019
INTERVENTIONS: Patients were randomly assigned either to the intervention group receiving coffee or the control group receiving tea. A total of $150 \mathrm{~mL}$ of the respective beverage was drunk 3 times per day every postoperative day until discharge.

MAIN OUTCOME MEASURES: The primary end point was time to first bowel movement. Secondary end points included the use of laxative, insertion of a nasogastric tube, length of hospital stay, and postoperative complications.

RESULTS: A total of 115 patients were randomly assigned: 56 were allocated to the coffee group and 59 to the tea group. After coffee intake, the first bowel movement occurred after a median of 65.2 hours versus 74.1 hours in the control group (intention-to-treat analysis; $p=$ 0.008). The HR for earlier first bowel movement after coffee intake was 1.67 ( $p=0.009)$. In the per-protocol analysis, hospital stay was shorter in the coffee group (6 d in the coffee group vs $7 \mathrm{~d}$ in the tea group; $p=0.043$ ).

LIMITATIONS: The rate of protocol violation, mostly coffee consumption in the tea arm, was relatively high, even if patients were clearly instructed not to consume coffee if they were in the tea arm.

CONCLUSIONS: Coffee intake after elective laparoscopic colorectal resection leads to faster recovery of bowel function. Therefore, coffee intake represents a simple and effective strategy to prevent postoperative ileus. See Video Abstract at http://links.lww.com/DCR/A955.

KEY WORDS: Coffee; Colorectal surgery; Enhanced recovery; Laparoscopy; Postoperative ileus. 


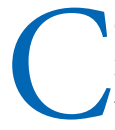
olorectal surgery, even performed by laparoscopy, is followed by a transient episode of GI hypomotility, which results in a postoperative ileus (POI). The usual duration of this benign postoperative situation varies between 3 and 5 days for colon surgery. ${ }^{1}$ A longer duration of POI will result in more postoperative complications, ${ }^{2}$ such as delayed surgical wound healing, atelectasis, pneumonia, and deep vein thrombosis ${ }^{3}$; in a prolonged hospital stay; and increased healthcare cost. ${ }^{3,4}$ Different studies reported that the duration of POI correlates with total surgery time, blood loss, total opiate dose, ${ }^{5}$ degree of surgical trauma, and bowel manipulation. ${ }^{6}$

Many attempts have been made to reduce the phase of postoperative intestinal hypomotility. The different strategies are well described in enhanced recovery and fast-track concepts and focus on minimal surgical trauma, rapid postoperative mobilization, early feeding, pre-emptive laxative treatment, restrictive fluid management, and minimal postoperative opioid prescription. ${ }^{7,8}$ Furthermore, several pharmacologic agents have been evaluated'; however, not all offered a convincing benefit, nor were they free of adverse effects.

Coffee is a worldwide highly consumed beverage, offering pleasure to many people of different cultures, and which many associate with an increase of bowel function. ${ }^{10}$ The aim of this study was to compare the time to first bowel movement after laparoscopic colorectal surgery between patients drinking coffee or tea postoperatively, in a randomized controlled trial (RCT).

\section{PATIENTS AND METHODS}

\section{Study Protocol}

The study was designed as a single-center, open-label RCT. Starting September 1, 2014, all of the eligible patients undergoing elective laparoscopic colon or rectal resection were considered for participation in the study unless exclusion criteria were present. Written informed consent was obtained 24 hours before surgery. The study protocol was approved and audited by the local ethics committee (study approval No. EKNZ: 2014-062; registration No. NCT02469441, http://www.clinicaltrials.gov).

Exclusion criteria were creation of a colostomy or ileostomy, any additional small bowel anastomosis, emergency operations, pre-existing ileus, preoperative radiation, known hypersensitivity or allergy to coffee, impaired mental state or language problems precluding informed consent, patients preoperatively taking agents influencing gut motility (eg, tricyclic antidepressants, opiods, or butylscopolamine), and postoperative epidural analgesia. Conversion to open surgery was not an exclusion criterion. Preoperative data, including BMI, comorbidities, medication, coffee drinking, and smoking habits were documented.
Patients were randomly assigned directly at the end of the operation in a 1:1 fashion to either receive coffee or tea. The randomization was assured with Randomizer (www. randomizer.at), a Web-based application for state-of-theart randomization within clinical trials, with stratification for indication and type of surgery (benign/malignant and right/left hemicolectomy). The study was a nonblinded RCT.

Patients in the treatment arm (coffee) received 1 cup of coffee 3 times per day $(150 \mathrm{~mL}$ at 8:00 $\mathrm{Am}, 12: 00 \mathrm{pM}$, and $5: 00 \mathrm{pM})$, in addition to the regular infusion therapy and/or alimentation starting the first day postoperatively. In the control arm, the same protocol was applied, replacing coffee with tea (excluding caffeine-containing tea, like black, green, and white tea) during the entire hospital stay.

All of the patients drank the same quantity and type of coffee coming from the same type of coffee machine. Coffee beans were taken from Graf Kaffee in BadenDättwil, Switzerland (P. Graf, Kaffeerösterei AG, 5405 Baden-Dättwil, Switzerland).

\section{Outcome Measures}

The primary end point was time to first bowel movement starting at the time of surgery completion. Included patients were given a diary and were asked (after instruction) to document on a daily basis if they had defecation or flatus and at what time; this was verified 3 times per day by the nurse. Secondary end points included the number of postoperative bowel movements until discharge, time to first flatus, use of laxatives, postoperative insertion of nasogastric tube, length of hospital stay, and postoperative complications (Dindo-Clavien classification) and were documented by the resident in charge of the patient. All of the patients were discharged with an appointment for a 30-day follow-up. S.H-G. and A.D. entered all of the data in the database.

\section{Perioperative Management}

To minimize bias, we defined a standard patient management protocol for the preoperative, perioperative, and postoperative phases of patients undergoing colorectal surgery.

Preoperatively, patients were allowed to eat 6 hours before surgery and to drink clear fluids 2 hours before surgery. An enema was applied on the evening before the operation and on the day of operation.

Perioperatively all of the operations were performed under general anaesthesia without additional local anaesthetic and without epidural analgesia. Antibiotic single-shot prophylaxis was always administered $(1.5 \mathrm{~g}$ of cefuroxime and $500 \mathrm{mg}$ of metronidazole intravenous (IV)). A urinary catheter was placed in the operating room. All of the left-sided anastomoses were circular stapled end to end. All of the right-sided anastomoses were handsewn 
side to side. Postoperative nausea and vomiting (PONV) prophylaxis was applied when the PONV Apfel score was $\geq 2$ (4 mg of mephenesin IV at induction and $1 \mathrm{mg}$ of droperidol IV at the end of surgery). In all of the patients the nasogastric tube was removed at the end of surgery.

Postoperatively, nonsteroidal anti-inflammatory analgesics (400 mg of Ibuprofen 3 times a day and $1 \mathrm{~g}$ of metamizole 4 times a day) formed the basis of the analgesic therapy. If additional analgesia was needed, $2 \mathrm{mg}$ of IV morphine was administered. If PONV was observed, patients first received $4 \mathrm{mg}$ of IV ondansetron, then $4 \mathrm{mg}$ of IV dexamethasone, and, when still persisting, $0.5 \mathrm{mg}$ of IV droperidol. The postoperative feeding regimen was standardized: on the day of surgery, patients were given 2 glasses of water, and solid food intake was started on the first postoperative day. The postoperative feeding regimen was determined by the patient's ability to consume food and was not dependent on intestinal function, such as passage of flatus or bowel movements. To not influence the primary outcome, no preemptive laxatives were given. However, if patients had no bowel movement until the fourth day, paraffin $3 \times 20 \mathrm{~mL}$ was administered orally. If there was no bowel movement on the fifth day, $50 \mathrm{~mL}$ of gastrographin were given orally. Postoperative mobilization was standardized and was the same for both groups. Criteria for hospital discharge included a clinical examination showing no evidence of complications, successful defecation, tolerance of solid meals, C-reactive protein $<100 \mathrm{mg} / \mathrm{L}$, no fever in the last 24 hours, pain visual analog scale score $\leq 4$, and completed full mobilization of the patient.

\section{Statistical Analysis}

\section{Sample Size}

The primary end point was time to first bowel movement. A sample size calculation was performed based on data of a previous RCT, including patients operated mainly by laparotomy and a different control group. In this previous trial, the mean reduction of postoperative time to first bowel movement was 13.6 hours, favoring the coffee group $(60.4( \pm 21.3 \mathrm{~h})$ vs $74.0 \mathrm{~h}( \pm 21.6 \mathrm{~h}) ; p=0.006) .{ }^{11}$ Based on these data, Monte-Carlo simulation revealed a group size of 46 patients to detect a relevant clinical and statistical difference, assuming an SD of $<22$ hours (2-sided significance level of $p \leq 0.05$; power of $80 \%)$. The expected minimum effect size (HR) was 1.23. Considering a possible dropout rate of $20 \%$ (protocol violations, withdrawal of informed consent), a total of 110 patients was calculated to be allocated to the trial interventions.

Cox proportional hazard regression analysis was used to analyze the primary end point, and data were plotted using a Kaplan-Meier estimator. Based on the multivariable Cox model, the estimated HR for the treatment effect, along with 95\% CI, was reported. Analyses were performed in an intention-to-treat (ITT) as well as in a per-protocol
(PP) setting, and $p$ values $\leq 0.05$ were considered statistically significant.

Similar to the primary end point, descriptive analyses of the secondary end points were performed in ITT and PP settings. Continuous variables were presented as median and interquartile range, and categorical variables as absolute numbers and percentage throughout the article. In univariate analysis, continuous variables were compared using Mann-Whitney $U$ test and categorical variables using the $\chi^{2}$ test or Fisher exact test, as appropriate. All $p$ values were calculated as 2 sided, and a $p \leq 0.05$ was considered statistically significant. All of the statistical analyses were performed using IBM SPSS Statistics version 22 for Macintosh (IBM Corp, Armonk, NY).

\section{RESULTS}

\section{Patient and Surgical Characteristics}

A total of 115 patients were correctly randomly assigned in the study between September 1, 2014, and December 31,2016 . Fifty-six patients were included in the intervention group (coffee), and 59 patients were included in the control group (tea). Figure 1 shows the CONSORT diagram. Baseline demographics and clinical/surgical characteristics are listed in Tables 1 and 2, and no significant differences between the 2 groups were found. A total of $88 \%$ of the patients kept the appointment for the 30-day follow-up, and the remaining patients were contacted by telephone. The 2 main indications for colorectal resections were diverticular disease and colon cancer.

Compliance to the protocol is shown in Table 3. These data show the effective coffee/tea intake (given 3 times per day to the patient) during the first 3 postoperative days.

\section{Effect of Coffee on First Bowel Movement}

The time to first bowel movement in the coffee group occurred after a median of 65.2 hours (95\% CI, 50.5-79.8 h) as compared with 74.1 hours (95\% CI, 60.7-87.5 h) in the control group (ITT analysis; $p=0.008$; Fig. 2). The HR for earlier first bowel movement after coffee intake was 1.67 (95\% CI, 1.14-2.44; $p=0.009$ ). Furthermore, $7.1 \%$ of the patients in the coffee group experienced the first bowel movement within 24 hours after surgery as compared with $1.7 \%$ in the tea group.

The ITT analysis showed no significant difference in length of hospital stay between the 2 groups. In the coffee arm, patients were discharged after a median of 6 days compared with 7 days in the tea group ( $p=0.13$; Table 4). In contrast, the PP analysis revealed a significant difference concerning length of hospital stay ( $6 \mathrm{~d}$ in the coffee group versus $7 \mathrm{~d}$ in the tea group; $p=0.043$; Table 5). There were no significant differences in time to first flatus, use of laxatives, or postoperative insertion of nasogastric tube (Tables 4 and 5). 


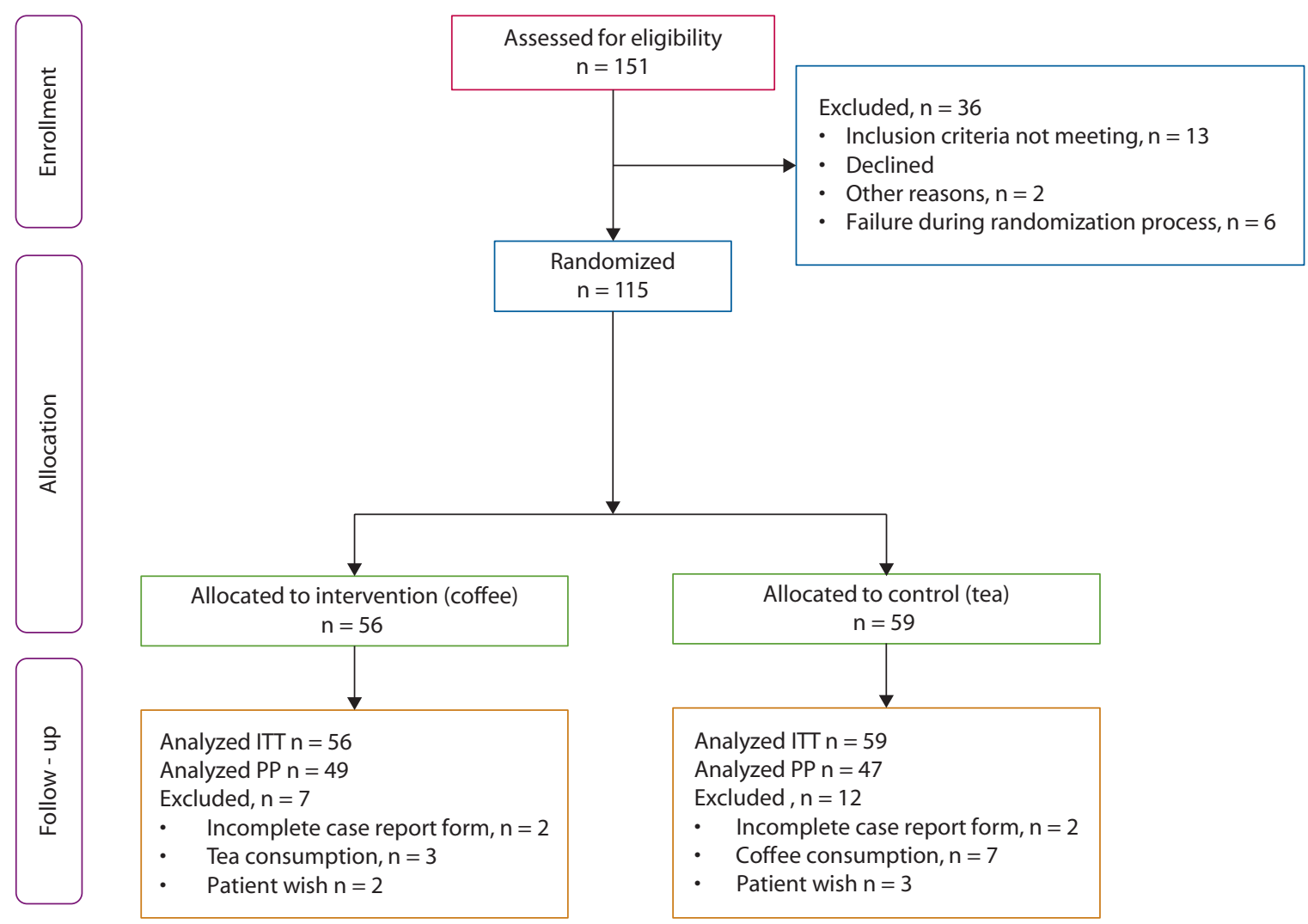

FIGURE 1. CONSORT diagram for the trial. ITT = intention-to-treat; PP = per-protocol.

\section{Perioperative Complications}

No deaths were observed in either group. The overall morbidity rate was $26.1 \%$, with no significant difference between the 2 groups. There was no significant difference concerning minor complications (Dindo-Clavien classification $<3 \mathrm{~b}$ ) or major complications (Dindo-Clavien classification $\geq 3$ b; Tables 4 and 5). Anastomotic leakage occurred twice in each group, representing $4 \%$ in coffee and $3 \%$ in the tea $\operatorname{arm}(p=1.00)$. Three of 4 anastomotic leaks required redo surgery corresponding with a grade $\mathrm{C}$ leak. Operative revisions were managed laparoscopically. One anastomotic leak was treated by antibiotics only (grade B). ${ }^{12}$

\section{DISCUSSION}

This RCT analyzes the effect of coffee intake on the time to first postoperative bowel movement after laparoscopic colorectal surgery. We show that early postoperative coffee intake leads to a significantly shorter time to first bowel movement and thus a potentially shorter length of hospital stay.

POI is a major focus of concern for surgeons because it increases postoperative morbidity, length of hospital stay, and thus healthcare costs. Different studies have shown that various factors such as minimally invasive surgery, narcotic sparing analgesia, restrictive fluid management, and early patient mobilization can help to reduce the length of POI. ${ }^{7}$ In enhanced recovery after surgery protocols, multimodal strategies have been described in which many of the above-mentioned factors are conjointly implemented during an enhanced postoperative phase. These protocols have proven their benefits but are not always easy to apply, because successful implementation requires dedicated support of different disciplines and professions, including anesthesia, pain specialists, nurses, physiotherapist, nutritionists, and surgeons. Prokinetic agents can also ameliorate the duration of POI, but their efficacy is not easy to predict, and adverse effects are relatively frequent. ${ }^{9}$ Therefore cheap, safe, and simple agents preventing or minimizing POI are needed.

Coffee is an enjoyable, inexpensive, and thus frequently consumed beverage in many households and is associated with increased bowel movements by many people. We therefore designed this RCT with the aim to investigate the effect of this highly consumed and appreciated beverage on bowel function after colorectal surgery. As a control, tea, another very popular beverage, was used.

The main finding was that coffee consumption shortens the time to the first postoperative bowel movement, occurring after a median of 65.2 hours in the coffee group and after 74.1 hours in the tea group. These results con- 
TABLE 1. Preoperative patient characteristics, intention to treat

\begin{tabular}{|c|c|c|}
\hline Variable & Coffee $(N=56)$ & Tea $(N=59)$ \\
\hline Age (range), y & $63(56-73)$ & $69(58-74)$ \\
\hline Men, n (\%) & $31(55)$ & $28(48)$ \\
\hline $\begin{array}{l}\text { Preoperative coffee intake } \\
\text { (range), cups per day }\end{array}$ & $2.5(1.6-3.5)$ & $2.0(1.0-3.5)$ \\
\hline Smoker, n (\%) & $15(27)$ & $11(19)$ \\
\hline BMI (range), $\mathrm{kg} / \mathrm{m}^{2}$ & $27.6(24.0-30.4)$ & $25.9(24.9-29.0)$ \\
\hline \multicolumn{3}{|l|}{ Comorbidities, n (\%) } \\
\hline Pulmonary & $9(16)$ & $3(5)$ \\
\hline Cerebral & $3(5)$ & $2(3)$ \\
\hline Vascular & $4(7)$ & $1(2)$ \\
\hline Hepatic & $1(2)$ & $4(7)$ \\
\hline Renal, & $4(7)$ & $4(7)$ \\
\hline Diabetes mellitus & $12(21)$ & $11(19)$ \\
\hline Hypertension & $20(36)$ & $16(27)$ \\
\hline Alcoholic disease & $0(0)$ & $0(0)$ \\
\hline Cardiac & $15(27)$ & $9(15)$ \\
\hline \multicolumn{3}{|l|}{ ASA grade, $\mathrm{n}(\%)$} \\
\hline I & $7(14)$ & $11(22)$ \\
\hline$\|$ & $36(69)$ & $34(67)$ \\
\hline III & $9(17)$ & $6(12)$ \\
\hline \multicolumn{3}{|l|}{ Diagnosis, n (\%) } \\
\hline Colonic cancer & $22(39)$ & $28(47)$ \\
\hline Diverticular disease I & $30(54)$ & $23(39)$ \\
\hline $\mathrm{IBD}$ & $0(0)$ & $1(2)$ \\
\hline Rectal cancer & $1(2)$ & $1(2)$ \\
\hline Other & $3(5)$ & $6(10)$ \\
\hline
\end{tabular}

cur with the findings from a previous randomized study testing the effect of coffee on POI as compared with warm water, in which time to the first bowel movement was also significantly shorter in the coffee arm. ${ }^{11}$ However, in this trial the large majority of included patients were operated by an open approach, and a majority additionally received an epidural anesthesia. Furthermore, coffee was

TABLE 2. Surgical procedures and perioperative results, intention to treat

\begin{tabular}{lcc} 
Variable & Coffee $(\mathrm{N}=56)$ & Tea $(\mathrm{N}=59)$ \\
\hline Surgical procedure, $\mathrm{n}(\%)$ & & \\
$\quad$ lleocecal resection & $0(0)$ & $1(2)$ \\
$\quad$ Right hemicolectomy & $15(27)$ & $21(35)$ \\
$\quad$ Left hemicolectomy & $8(14)$ & $6(10)$ \\
$\quad$ Sigmoid resection & $30(54)$ & $30(51)$ \\
$\quad$ Rectal resection & $3(5)$ & $1(2)$ \\
Surgical approach, $\mathrm{n}(\%)$ & & $3(5)$ \\
$\quad$ Converted & $1(2)$ & $56(95)$ \\
$\quad$ Laparoscopic & $55(98)$ & $150(130-180)$ \\
Duration of operation & $160(136-185)$ & \\
$\quad$ (range), min & & $50(10-100)$ \\
Blood loss (range), $\mathrm{mL}$ & $50(10-100)$ & $0(0-0)$ \\
Packed red blood cells & $0(0-0)$ & 1500 \\
$\quad$ (range), bag & & $\mathrm{v}(1000-1900)$ \\
Infusion volume & & \\
intraoperatively & $(1600$ & $2(3)$ \\
$\quad$ (range), mL & $(1300-2075)$ & \\
Patients on ICU & & \\
postoperatively, $\mathrm{n}(\%)$ & $2(4)$ & \\
\hline ICU = intensive care unit. & &
\end{tabular}

$\mathrm{ICU}=$ intensive care unit.
TABLE 3. Compliance to the protocol

\begin{tabular}{|c|c|c|c|}
\hline Day & $\begin{array}{c}\text { Coffee } \\
(N=49), \\
n(\%)\end{array}$ & $\begin{array}{c}\text { Tea }(N=47), \\
n(\%)\end{array}$ & $p$ \\
\hline \multicolumn{4}{|l|}{ POD 1} \\
\hline Morning & $43(87.8)$ & 47 (100) & 0.03 \\
\hline Afternoon & 44 (89.8) & 41 (87.2) & 0.76 \\
\hline Evening & 38 (77.6) & $41(87.2)$ & 0.29 \\
\hline \multicolumn{4}{|l|}{ POD 2} \\
\hline Morning & $48(98.0)$ & 47 (100) & 1.00 \\
\hline Afternoon & 46 (93.9) & 46 (97.9) & 0.62 \\
\hline Evening & $36(73.5)$ & 46 (97.9) & $<0.01$ \\
\hline \multicolumn{4}{|l|}{ POD 3} \\
\hline Morning & 45 (91.8) & 46 (97.9) & 0.36 \\
\hline Afternoon & 44 (89.8) & 46 (97.9) & 0.20 \\
\hline Evening & $38(77.6)$ & 41 (87.2) & 0.29 \\
\hline
\end{tabular}

Data show effective coffee/tea intake (given 3 times per day to the patient) during the first 3 postoperative days (POD).

compared with a glass of warm water. Because tea is one of the world's most consumed beverages,$^{13}$ we believe that its comparison to coffee reflects more accurately the postoperative in-hospital situation.

Our PP analysis showed a significantly shorter hospital stay in the coffee group ( $6 \mathrm{~d}$ in the coffee group versus $7 \mathrm{~d}$ in the tea group). Therefore, early postoperative coffee consumption might also help to reduce the length of hospital stay, because a larger study population, based on a power calculation to detect a difference in the duration of hospital stay, could reveal a significant difference also in an ITT analysis.

Prokinetic agents reducing the postoperative intestinal paralytic phase are frequently evaluated because of their potential high impact on patient well-being and hospital costs. Drugs such as metoclopramide, erythromycin, lidocaine, and neostigmine have shown ambiguous results on POI, and some can entail severe adverse effects. ${ }^{9} \mathrm{Al}-$ though alvimopan has shown a positive response, ${ }^{9}$ it is not approved for use in Switzerland. Chewing gum acts in a vagal cholinergic stimulation of the GI tract, similar to oral food intake but with theoretically less risk of vomiting and aspiration. A meta-analysis of 7 clinical trials could demonstrate that chewing gum significantly reduced the time to first flatus and the time to first passage of feces. ${ }^{14}$ However, chewing gum is not widely appreciated, and the latest large RCT failed to show a benefit of chewing gum on the length of hospital stay and the time to first defection. ${ }^{15}$

Coffee is a universally popular beverage acting as a prokinetic agent in our setting of laparoscopic colorectal resections. Although the difference of 9 hours on earlier bowel movement might seem discrete, it can nevertheless lead to a quicker hospital discharge, especially in patients with an uneventful postoperative course, thus representing a clear benefit in the light of optimized colorectal pathways. 
Intention-to-treat analysis

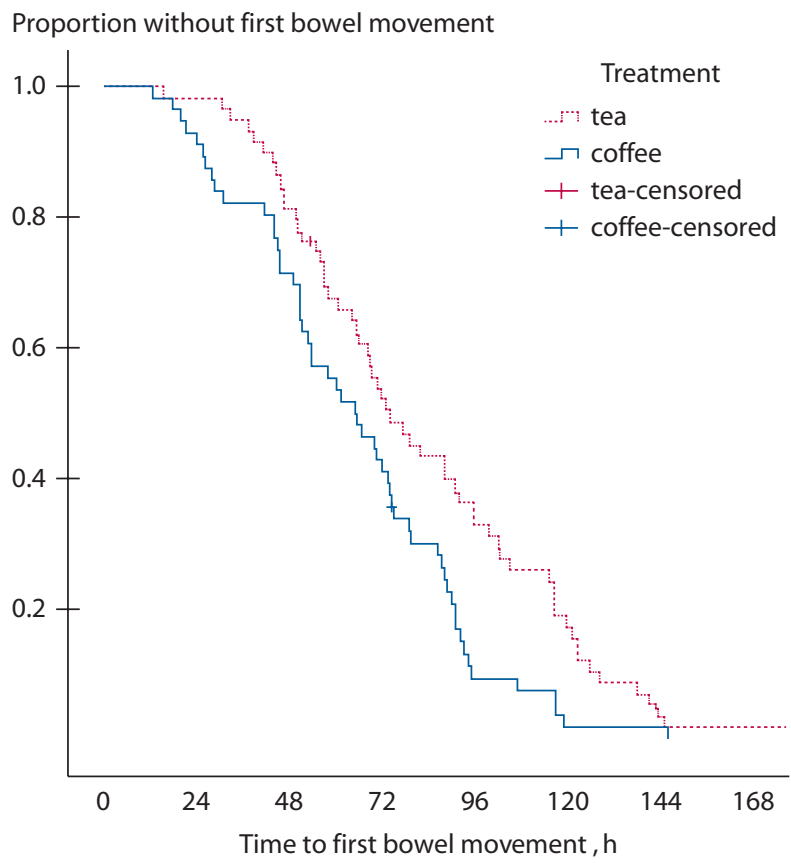

FIGURE 2. Kaplan-Meier plot: time to first bowel movement.

Although coffee has been shown previously to increase colon activity, the precise mechanism of intestinal motility acceleration remains unknown. ${ }^{16}$ Caffeine induces excitatory effects on the nervous and cardiovascular systems. ${ }^{17}$

\section{TABLE 4. Outcome data in intention-to-treat analysis}

\begin{tabular}{lccc} 
Variable & Coffee $(\mathrm{N}=56)$ & Tea $(\mathrm{N}=59)$ & $p$ \\
\hline Time to first bowel movement & 65.2 & 74.1 & 0.008 \\
(95\% Cl), h & $(50.5-79.8)$ & $(60.7-87.5)$ & \\
No. of bowel movements until & $4(3-6)$ & $4(3-6)$ & 0.76 \\
discharge (range) & & & \\
Time to first flatus (range), h & $40(29-52)$ & $31(25-46)$ & 0.08 \\
Use of any laxative, $\mathrm{n}(\%)$ & $16(29)$ & $24(41)$ & 0.24 \\
Postoperative insertion of & $3(6)$ & $3(5)$ & 1.00 \\
NGT, $\mathrm{n}(\%)$ & & & \\
Length of hospital stay & $6(5-9)$ & $7(6-8)$ & 0.14 \\
(range), d & & & \\
Total morbidity (Dindo-Clavien & & & \\
classification) & & & \\
Any complication, $\mathrm{n}(\%)$ & $15(27)$ & $15(25)$ & 1.00 \\
Minor complications (<3b) & $12(21)$ & $9(15)$ & 0.47 \\
Major complications $(\geq 3 \mathrm{~b})$ & $3(5)$ & $6(10)$ & 0.49 \\
I & $5(9)$ & $4(7)$ & 0.74 \\
II & $7(13)$ & $4(7)$ & 0.36 \\
IIIA & $0(0)$ & $1(2)$ & 1.00 \\
IIIB & $3(5)$ & $4(7)$ & 0.52 \\
IVA & $0(0)$ & $1(2)$ & 1.00 \\
IVB & $0(0)$ & $1(2)$ & 1.00 \\
V & $0(0)$ & $0(0)$ & 1.00 \\
Anastomotic leakage, $\mathrm{n}(\%)$ & $2(4)$ & $2(3)$ & 1.00 \\
Grade A & $0(0)$ & $0(0)$ & 1.00 \\
Grade B & $1(2)$ & $0(0)$ & 0.49 \\
Grade C & $1(2)$ & $2(3)$ & 1.00 \\
\hline
\end{tabular}

NGT = nasogastric tube.
Per-protocol analysis

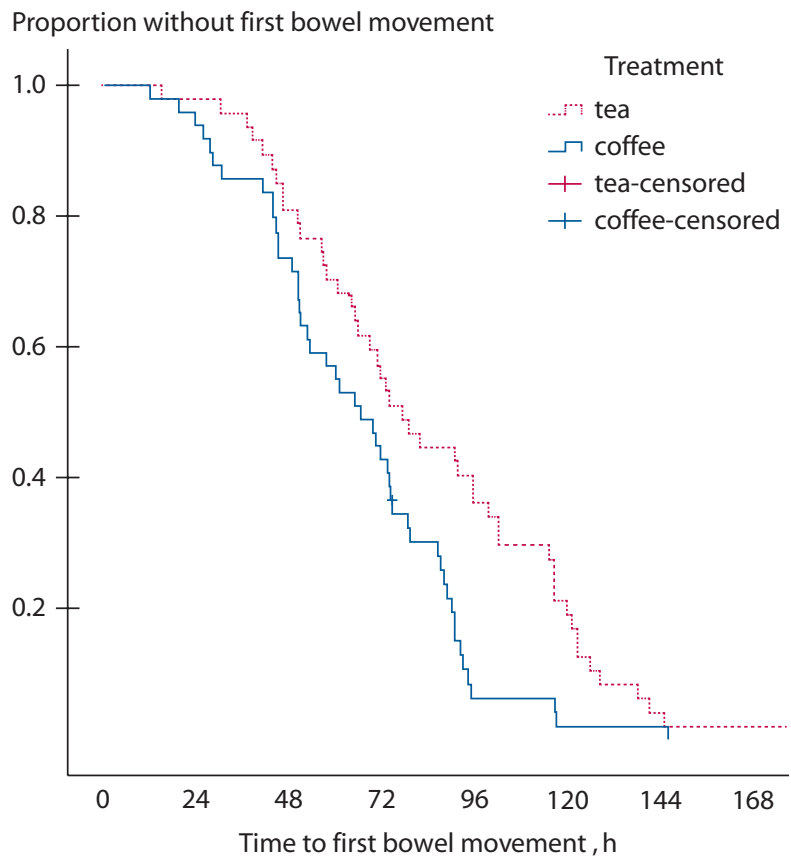

Therefore, caffeine was hypothesized to be the primary mediator of intestinal motility acceleration. ${ }^{17}$ However, studies comparing regular and decaffeinated coffee have shown equal or better effects on intestinal motility for decaffeinated coffee. ${ }^{10,18}$ In addition, the role of coffee habituation, that is, whether patients with a high coffee consumption experience a lower impact on GI function, is also unknown. We found none of the patient-related factors, including preoperative habitual coffee intake, to have a significant impact on postoperative bowel activity. Along this line, the effect of tea on intestinal motility remains unknown as well. Tea was hypothesized to exert a constipating effect through a theophylline-mediated (a metabolite from caffeine), extracellular dehydration. ${ }^{19}$ On the other hand, a stimulating effect of tea on GI motility has also been reported. ${ }^{20}$ Therefore, in our study caffeinecontaining tea was excluded.

Apart from the effectiveness, the frequency of adverse effects and complication rates are crucial factors in determining the efficacy of a new agent. We found no significant differences between coffee- and tea-drinking patients. Our observed overall complication rate of $26.1 \%$ is in line with other reports. ${ }^{11,21}$

The strength of this study lies in the nature of RCTs. Powered to detect a difference in time to first postoperative bowel movement after colorectal resections, the conclusion that emerged is secure and generalizable. Even after a laparoscopic approach, early postoperative coffee intake exerts a beneficial effect on bowel function. The easy implementation in daily hospital practice, its nearly 
TABLE 5. Outcome data in PP analysis

\begin{tabular}{|c|c|c|c|}
\hline \multirow{2}{*}{$\begin{array}{l}\text { Variable } \\
\text { Time to first bowel movement } \\
(95 \% \mathrm{Cl}), \mathrm{h}\end{array}$} & \multicolumn{2}{|c|}{ Coffee $(N=49)$ Tea $(N=47)$} & \multirow{2}{*}{$\begin{array}{c}p \\
0.006\end{array}$} \\
\hline & $\begin{array}{c}66.8 \\
(52.7-81.0)\end{array}$ & $\begin{array}{c}77.3 \\
(63.8-90.9)\end{array}$ & \\
\hline $\begin{array}{l}\text { No. of bowel movements until } \\
\text { discharge (range) }\end{array}$ & $4(2-5)$ & $4(3-6)$ & 0.50 \\
\hline Time to first flatus (range), $\mathrm{h}$ & $40(28-51)$ & $30(25-45)$ & 0.05 \\
\hline Use of any laxative, n (\%) & $16(33)$ & $23(49)$ & 0.15 \\
\hline $\begin{array}{l}\text { Postoperative insertion } \\
\text { of NGT, } n(\%)\end{array}$ & $3(6)$ & $3(6)$ & 1.00 \\
\hline $\begin{array}{l}\text { Length of hospital stay } \\
\text { (range), d }\end{array}$ & $6(5-8)$ & $7(6-8)$ & 0.043 \\
\hline \multicolumn{4}{|l|}{$\begin{array}{l}\text { Total morbidity (Dindo-Clavien } \\
\text { classification) }\end{array}$} \\
\hline Any complication, n (\%) & $13(23)$ & $9(15)$ & 0.47 \\
\hline Minor complications (<3b) & $10(18)$ & $6(10)$ & 0.41 \\
\hline Major complications ( $\geq 3 \mathrm{~b}$ ) & $3(5)$ & $3(5)$ & 1.00 \\
\hline I & $4(8)$ & $2(4)$ & 0.68 \\
\hline II & $6(12)$ & $3(6)$ & 0.49 \\
\hline IIIA & $0(0)$ & $1(2)$ & 0.49 \\
\hline IIIB & $3(6)$ & $2(4)$ & 1.00 \\
\hline IVA & $0(0)$ & $0(0)$ & 1.00 \\
\hline IVB & $0(0)$ & $1(2)$ & 0.49 \\
\hline V & $0(0)$ & $0(0)$ & 1.00 \\
\hline Anastomotic leakage, n (\%) & $2(2)$ & $2(3)$ & 1.00 \\
\hline Grade A & $0(0)$ & $0(0)$ & 1.00 \\
\hline Grade B & $1(2)$ & $0(0)$ & 1.00 \\
\hline Grade C & $1(2)$ & 2(3) & 1.00 \\
\hline
\end{tabular}

NGT = nasogastric tube.

nonexisting adverse effects, and low cost make postoperative coffee intake even more attractive. The compliance to the protocol was high in both groups. At 2 time points, patients were slightly less compliant in the coffee group compared with the tea group, which could point to an even stronger effect of coffee.

However, a few limitations of our study deserve consideration. The rate of protocol violation (mostly coffee consumption in the tea arm), was relatively high (16.9\%). Because a specific, commonly accepted definition of POI is still lacking and an accurate diagnostic modality to differentiate POI from PONV is not yet available, ${ }^{22}$ we could only use a clearly assessable surrogate parameter, first postoperative bowel movement, as an end point for our RCT. Therefore, the observed positive effect of coffee might not translate to other symptoms of POI.

Although not statistically significant, more major complications were observed in the tea as compared with the coffee group. Although this could have an impact on POI, time to first defecation was not significantly longer in most of the cases with major complications as compared with the other patients in the tea group (data not shown). Furthermore, no association between postoperative tea intake and a higher complication rate after laparoscopic colorectal resections is known. Therefore, by increasing the sample size, an adjustment of the difference in major complications between the 2 groups could be expected.

\section{CONCLUSION}

Our study shows that early postoperative coffee intake after elective laparoscopic colorectal resection leads to a faster recovery of bowel function and thus potentially decreases length of hospital stay. Because of its easy availability and low adverse effects, coffee could be integrated in the postoperative management of patients undergoing colorectal resections.

\section{REFERENCES}

1. Livingston EH, Passaro EP Jr. Postoperative ileus. Dig Dis Sci. 1990;35:121-132.

2. Grass F, Slieker J, Jurt J, et al. Postoperative ileus in an enhanced recovery pathway-a retrospective cohort study. Int J Colorectal Dis. 2017;32:675-681.

3. Senagore AJ. Pathogenesis and clinical and economic consequences of postoperative ileus. Am J Health Syst Pharm. 2007;64(20 suppl 13):S3-S7.

4. Boeckxstaens GE, de Jonge WJ. Neuroimmune mechanisms in postoperative ileus. Gut. 2009;58:1300-1311.

5. Artinyan A, Nunoo-Mensah JW, Balasubramaniam S, et al. Prolonged postoperative ileus-definition, risk factors, and predictors after surgery. World J Surg. 2008;32:1495-1500.

6. Türler A, Moore BA, Pezzone MA, Overhaus M, Kalff JC, Bauer AJ. Colonic postoperative inflammatory ileus in the rat. Ann Surg. 2002;236:56-66.

7. Kehlet H, Dahl JB. Anaesthesia, surgery, and challenges in postoperative recovery. Lancet. 2003;362:1921-1928.

8. Schwenk W. Fast track rehabilitation in visceral surgery [in German]. Chirurg. 2009;80:690-701.

9. Traut U, Brügger L, Kunz R, et al. Systemic prokinetic pharmacologic treatment for postoperative adynamic ileus following abdominal surgery in adults. Cochrane Database Syst Rev. 2008; (1):CD004930.

10. Brown SR, Cann PA, Read NW. Effect of coffee on distal colon function. Gut. 1990;31:450-453.

11. Müller SA, Rahbari NN, Schneider F, et al. Randomized clinical trial on the effect of coffee on postoperative ileus following elective colectomy. Br J Surg. 2012;99:1530-1538.

12. Rahbari NN, Weitz J, Hohenberger W, et al. Definition and grading of anastomotic leakage following anterior resection of the rectum: a proposal by the International Study Group of Rectal Cancer. Surgery. 2010;147:339-351.

13. Stone D. The world's top drink. Natl Geogr Mag. https:// www.nationalgeographic.com/people-and-culture/onward/2014/04/28/the-worlds-top-drink/. Accessed April 3, 2019.

14. Vásquez W, Hernández AV, Garcia-Sabrido JL. Is gum chewing useful for ileus after elective colorectal surgery? A systematic review and meta-analysis of randomized clinical trials. J Gastrointest Surg. 2009;13:649-656.

15. de Leede EM, van Leersum NJ, Kroon HM, van Weel V, van der Sijp JRM, Bonsing BA; Kauwgomstudie Consortium. Multicentre randomized clinical trial of the effect of chewing gum after abdominal surgery. Br J Surg. 2018;105:820-828.

16. Rao SS, Welcher K, Zimmerman B, Stumbo P. Is coffee a colonic stimulant? Eur J Gastroenterol Hepatol. 1998;10:113-118. 
17. Boekema PJ, Samsom M, van Berge Henegouwen GP, Smout AJ. Coffee and gastrointestinal function: facts and fiction-a review. Scand J Gastroenterol Suppl. 1999;230:35-39.

18. Dulskas A, Klimovskij M, Vitkauskiene M, Samalavicius NE. Effect of coffee on the length of postoperative ileus after elective laparoscopic left-sided colectomy: a randomized, prospective single-center study. Dis Colon Rectum. 2015;58:1064-1069.

19. Højgaard L, Arffmann S, Jørgensen M, Krag E. Tea consumption: a cause of constipation? Br Med J (Clin Res Ed). 1981;282:864.
20. Chaudhuri L, Basu S, Seth P, et al. Prokinetic effect of black tea on gastrointestinal motility. Life Sci. 2000;66:847-854.

21. Leichtle SW, Mouawad NJ, Welch KB, Lampman RM, Cleary RK. Risk factors for anastomotic leakage after colectomy. Dis Colon Rectum. 2012;55:569-575.

22. Gero D, Gié O, Hübner M, Demartines N, Hahnloser D. Postoperative ileus: in search of an international consensus on definition, diagnosis, and treatment. Langenbecks Arch Surg. 2017;402:149-158. 